\title{
Diferencias en la prevalencia de adenomas avanzados y carcinomas colorrectales según edad y sexo
}

Prevalence of advanced adenomas and colorectal cancer according to age and sex

Ferlitsch M, y col. JAMA. 2011;306(12):1352-8.

\section{Objetivo}

Determinar y comparar la prevalencia y número necesario para rastrear* (NNR) de adenomas, adenomas avanzados y carcinomas colorrectales (CCR) para diferentes grupos etarios en hombres y mujeres.

\section{Diseño, lugar y pacientes}

Estudio descriptivo de corte transversal ${ }^{*}$, realizado en Austria. Se incluyeron 44.350 participantes (22.598 mujeres, 21.752 hombres) de un programa nacional de rastreo de CCR con colonoscopía durante un período de cuatro años (2007 a 2010).

\section{Descripción del procedimiento diagnóstico}

Un organismo central recibió los informes de todas las colonoscopías y análisis histopatológicos, categorizados a su vez de acuerdo a la lesión más avanzada identificada. Se consideró adenomas avanzados a aquellos que tuviesen alto grado de displasia, compo- nente velloso o tubulovelloso en la histopatología, o adenomas tubulares de diámetro mayor o igual a $10 \mathrm{~mm}$.

Medición de resultados principales

Prevalencia y NNR de adenomas, adenomas avanzados y CCR en diferentes grupos etarios de hombres y mujeres.

\section{Resultados principales}

Los hallazgos macroscópicos incluyeron: pólipos $34,4 \%$ $(n=15.267)$, cáncer de colon $0,4 \%(n=162)$, cáncer de recto $0,2 \%(n=92)$, otros diagnósticos $3,6 \%(n=1.600)$. El $61,4 \% \quad(n=$ 27.212) de las colonoscopías fueron normales. Los resultados principales se resumen en la tabla 1.

El género masculino se asoció con mayor prevalencia de adenomas (OR* 1,9 IC95\% 1,8 a 2,0), adenomas avanzados (OR 1,8 [IC95\% 1,6 a 1,9), y CCR (OR 2,1 IC95\% 1,7 a 2,5).

Tabla 1. Prevalencia y NNR de adenomas avanzados y cáncer colorrectal en hombres y mujeres entre los 45 y 75 años

\begin{tabular}{|c|c|c|c|c|}
\hline \multirow[b]{3}{*}{ Grupo etario } & \multicolumn{4}{|c|}{ Adenoma Avanzado } \\
\hline & \multicolumn{2}{|c|}{ Mujeres } & \multicolumn{2}{|c|}{ Hombres } \\
\hline & Prevalencia, n (\%) [IC 95\%] & NNR [IC 95\%] & Prevalencia, n (\%) [IC 95\%] & NNR [IC 95\%] \\
\hline Global & $1050(4,65)[4,4$ a 4,9$]$ & $21,5[20,3$ a 22,8$]$ & $1731(9,76)[7,6$ a 8,3] & $12,6[12,0$ a 13,2$]$ \\
\hline \multicolumn{5}{|l|}{ Edad, a } \\
\hline 45 a 49 & $14(3,5)[1,5$ a 5,8$]$ & $28,8[17,4,1$ a 52,3$]$ & $17(3,8)[2,3$ a 6,1$]$ & $26,1[16,5$ a 44,4$]$ \\
\hline 50 a 54 & $155(2,9)[2,5$ a 3,4$]$ & $34,0[29,1$ a 40,0$]$ & $263(5,0)[4,4$ a 5,6$]$ & $20,0[17,8$ a 22,6$]$ \\
\hline 55 a 59 & $174(3,9)[3,3$ a 4,5$]$ & $26,0[22,5$ a 30,2$]$ & $301(7,0)[6,3$ a 7,9$]$ & $14,2[12,7$ a 15,9$]$ \\
\hline 60 a 64 & $219(5,1)[4,4$ a 5,8$]$ & $19,7[17,3$ a 22,5$]$ & $355(9,0)[8,2$ a 10$]$ & $11,0[10,0$ a 12,2$]$ \\
\hline 65 a 69 & $241(6,0)[5,3$ a 6,8$]$ & $16,6[14,7$ a 18,9$]$ & $401(10,3)[9,4$ a 11,3$]$ & $9,7[8,8$ a 10,7$]$ \\
\hline \multirow[t]{2}{*}{70 a 74} & $114(5,6)[4,7$ a 6,7$]$ & $17,8[14,9$ a 21,5$]$ & $212(10,4)[9,1$ a 11,8$]$ & $9,7[8,5$ a 11,0$]$ \\
\hline & \multicolumn{4}{|c|}{ Cáncer Colorrectal } \\
\hline Grupo etario & 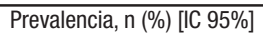 & NNR [IC 95\%] & Prevalencia, n (\%) [IC 95\%] & NNR [IC 95\%] \\
\hline Global & $165(0,7)[0,62$ a 0,85$]$ & $137,0[117,7$ a 160,4$]$ & $326(1,5)[1,3$ a 1,7$]$ & $66,7[60,0$ a 74,6$]$ \\
\hline \multicolumn{5}{|l|}{ Edad, a } \\
\hline 45 a 49 & $1(0,2)[0,01$ a 1,4$]$ & $403,0[72,7$ a 15918,2$]$ & $5(1,1)[0,4$ a 2,6$]$ & $88,6[38,3$ a 272,1$]$ \\
\hline 50 a 54 & $20(0,4)[0,2$ a 0,6$]$ & $263,6[170,8$ a 431,2$]$ & $42(0,8)[0,6$ a 1,1$]$ & $125,3[92,8$ a 173,9$]$ \\
\hline 55 a 59 & $26(0,6)[0,4$ a 0,8$]$ & $173,8[118,8$ a 265,8$]$ & $57(1,3)[1$ a 1,7$]$ & $75,0[58,0$ a 98,9$]$ \\
\hline 60 a 64 & $32(0,7)[0,5$ a 1$]$ & $135,0[95,8$ a 197,1$]$ & $52(1,3)[1$ a 1,7$]$ & $75,4[57,6$ a 100,8$]$ \\
\hline 65 a 69 & $49(1,2)[0,9$ a 1,6$]$ & $81,8[62,0$ a 110,4$]$ & $72(1,9)[1,5$ a 2,3$]$ & $53,9[42,9$ a 68,8$]$ \\
\hline 70 a 74 & $15(0,7)[0,4$ a 1,2$]$ & $135,4[82,3$ a 241,6$]$ & $39(1,9)[1,4$ a 2,6$]$ & $52,5[38,6$ a 73,7$]$ \\
\hline
\end{tabular}

\section{Conclusiones}

En una cohorte de individuos Austríacos sometidos a rastreo con colonoscopía durante cuatro años, la prevalencia y NNR de adenomas avanzados fue comparable entre hombres de 45 a 49 años y mujeres

de 55 a 59 años, mientras que el riesgo de CCR fue similar entre hombres de 55 a 59 años y mujeres de 65 a 69 años.

Fuentes de financiamiento: Fondo para Controles Preventivos y Promoción de la Salud a través de la Asociación Principal de Instituciones Austríacas de Seguridad Social.

\section{Comentario}

Este estudio se suma a otros publicados con anterioridad ${ }^{12,3,4}$ en los que se ha descripto un mayor riesgo de neoplasia colo-rrectal avanzada en los hombres con respecto a las mujeres de la misma edad. Los autores sugieren que el sexo masculino es un factor de riesgo independiente que debería ser considerado por las guías de práctica clínica, cuyas recomendaciones actuales de rastreo son independientes de género a partir de los 50 años ${ }^{5.67}$. Algunos investigadores han sugerido que una diferenciación apropiada en la edad de inicio del rastreo de acuerdo al sexo podría ser tan relevante como la historia familiar desde un punto de vista de la salud pública², e incluso existe evidencia de que el género masculino y el tabaquismo tienen mayor efecto en la prevalencia de CCR que los antecedentes familiares'.
Como limitaciones metodológicas se destacan el riesgo de sesgo en los resultados de los pacientes menores de 50 años debido al escaso número de observaciones, y la falta de ajuste por otros posibles factores confundidores (como por ej. historia familiar de CCR, tabaquismo, obesidad).

\section{Conclusiones del comentador}

Aunque existe evidencia que sugiere que la estratificación del riesgo para iniciar el rastreo de CCR debería incluir consideraciones de edad específicas de género, aún se necesitan estudios que demuestren la eficacia clínica del rastreo a diferentes edades.

Valeria Vietto [ Servicio de Medicina Familiar y Comunitaria del Hospital Italiano de Buenos Aires. valeria.vietto@ hospitalitaliano.org.ar ] Ver glosario Vietto V. Diferencias en la prevalencia de adenomas avanzados y carcinomas colorrectales según edad y sexo. Evid Act Pract Ambul Oct-Dic 2012;15(4):132. Comentado de: Ferlitsch M, Reinhart K, Pramhas S, y col. Sex-specific prevalence of adenomas, advanced adenomas, and colorectal cancer in individuals undergoing screening colonoscopy. JAMA. 2011 sep 28;306(12):1352-8.

Referencias bibliográficas

1. Regula J, y col. Colonoscopy in colorectal-cancer screening for detection of advanced neoplasia. N. Engl. J. Med. 2006 nov 2;355(18):1863-72.

2. Brenner $\mathrm{H}$, y col. Gender differences in colorectal cancer: implications for age at initiation of screening. Br. J. Cancer. 2007 mar 12;96(5):828-31

3. Rundle AG, y col. Colonoscopic screening in average-risk individuals ages 40 to 49 vs 50 to 59 years. Gastroenterology. 2008 may;134(5):1311-5.

4. Brenner $\mathrm{H}, \mathrm{y}$ col. Risk of progression of advanced adenomas to colorectal cancer by age and sex: estimates based on 840,149 screening colonoscopies. Gut. 2007 nov;56(11):1585-9.

5. Screening for colorectal cancer: U.S. Preventive Services Task Force recommendation statement. Ann. Intern. Med. 2008 Nov 4;149(9):627-637.

6. Whitlock EP, y col. Screening for colorectal cancer: a targeted, updated systematic review for the U.S. Preventive Services Task Force. Ann. Intern. Med. 2008 Nov 4;149(9):638-658

7. Levin B, y col. Screening and surveillance for the early detection of colorectal cancer and adenomatous polyps, 2008: a joint guideline from the American Cancer Society, the US Multi-Society Task Force on Colorectal Cancer, and the American College of Radiology. Gastroenterology. 2008 May;134(5):1570-1595.

8. Hoffmeister M, y col. Male sex and smoking have a larger impact on the prevalence of colorectal neoplasia than family history of colorectal cancer. Clin. Gastroenterol. Hepatol. 2010 oct;8(10):870-6. 